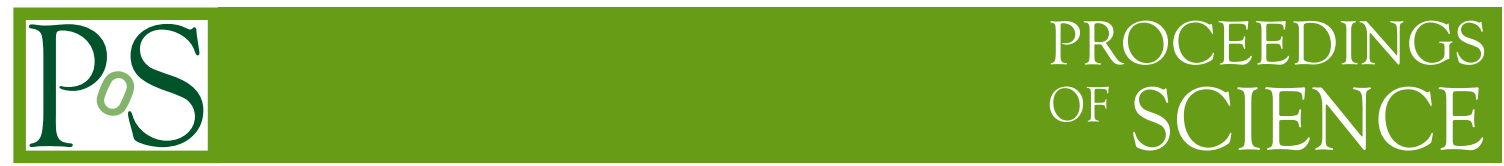

\title{
Dark Matter 2017
}

\section{Giulio Auriemma*†}

INFN Sezione di Roma, Roma, Italy

Cosmology and astronomical observation of distant galaxies have given in the last years an overwhelming evidence of the existence of dark matter in the Universe, but have taught very little about its physical nature. This paper is a short review of the present status of the experimental investigations of non-gravitational interaction of this elusive kind of matter with the ordinary one.

XII Multifrequency Behaviour of High Energy Cosmic Sources Workshop

12-17 June, 2017

Palermo, Italy

*Speaker.

†e-mail:Giulio.Auriemma@ cern.ch 


\section{Introduction}

In the last year $\sim 840$ papers have been uploaded to the "physics" section of the arXiv database, with the words "dark matter" in the title. Most of these are already published on refereed scientific journals. In Fig. 1 is shown my personal interpretation of the breakdown in the various the field of that papers, based on the title or abstract. About $35 \%$ of them discuss possible alternative interpretations, respect to the standard assumptions, namely WIMP, axions, etc. It is worth noticing that about $20 \%$ of the papers deal with different techniques for the investigation of the nature of the particles.

In the following we will discuss in $\S 1 \S 2$

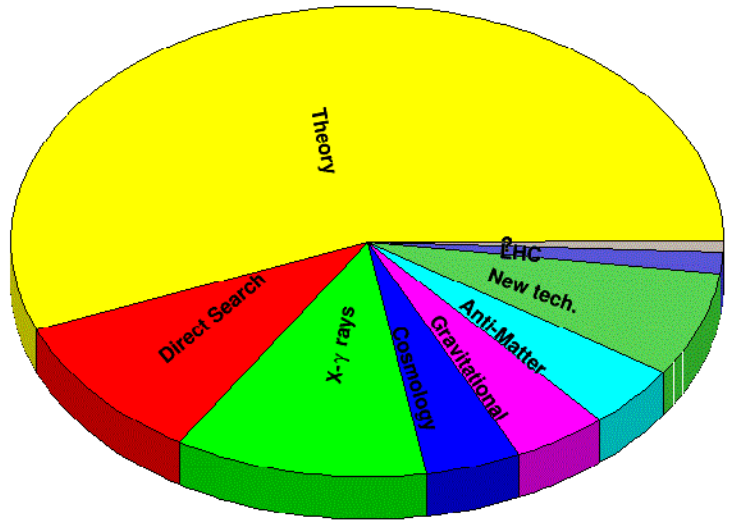

Figure 1: Partition of the papers on dark matter published from June 2016 to May 2017.

the present status of the search for scattering of the DM with matter in detectors, namely the so called "direct searches" experiments, in $\S 3$ the search of new types of particles produced in multi$\mathrm{TeV} p p$ collisions at LHC. In $\S 4$ of the paper we will illustrate some recent experimental results on very heavy multiquarks states (with mass $\sim 4.4 \mathrm{GeV} / c^{2}$ ), that are copiously produced at LHC, and in the quark-gluon plasma phase of the primordial universe.

The understanding of the physical nature of dark matter is in this moment particularly weak! The only clear information that we have about DM is that very likely it is there and interacts gravitationally with the ordinary matter.

In the following we will discuss in $\$ 2$ what we know about the DM from cosmology, in $\S 3$ the status of the searches for cosmological DM in galactic halos by the production of astrophysical $\gamma$-rays, in $\S 4$ direct search for scattering of the DM in underground detectors, In $\$ 5$ the search for new types of particles produced in multi-TeV $p p$ collisions at LHC and the kind of information that we have on the DM particles. Finally in $\S 6$ of the paper we discuss briefly the recent experimental results on very heavy multiquarks states (with mass $\sim 4.4 \mathrm{GeV} / c^{2}$ ), that are copiously produced at LHC, and in the quark-gluon plasma phase of the primordial universe.

\section{Dark Matter in Cosmology}

The Dark Matter (DM) has been discovered by Zwicky[1,2] in clusters of galaxies, more than 80 years ago. An important confirmation was given by Rubin and collaborators[3], around 50 years ago, that the rotational mass of the halo of the nearest galaxy, M 31, was ten times larger that the luminosity mass. The cosmological evidence for dark matter becomes everyday stronger from the results of the study of the Cosmic Microwave Background (CMBR) with the Planck satellite[4]. The more recent fit of the CMBR small scale fluctuations favors a flat Lambda Cold Dark Matter Model $(\Lambda \mathrm{CDM})$, with about $30 \%$ of DM with an absolute density in the present Universe: 


$$
\rho_{\chi}=(3 . \pm 0.3) \times 10^{-6} \mathrm{GeV} / \mathrm{c}^{2} \mathrm{~cm}^{-3}
$$

The density ratio of the CDM to the ordinary matter is $\rho_{\chi} / \rho_{b}=52.5 \pm 5$. It is to remember that in the technical language of experimental cosmology the adjective "Cold" indicates a component of the primordial plasma non-relativistic and non-collisional at $z_{e q}$. In practice, observed small-scale anisotropy of the CMBR [5], suggests the weak limit $\sigma / m \leq 1.5 \times 10^{-26} \mathrm{~cm}^{2} / \mathrm{GeV} / \mathrm{c}^{2}$.

If not formed by Majorana fermions singlets, the CDM has been in thermal equilibrium with the background radiation in the primordial universe when $T_{\gamma} \gg m_{\chi}$. In this early period the expansion of the universe the temperature decrease $T_{\gamma} \propto 1 / \sqrt{t_{U}}$. For WIMPs with mass $m_{\chi} \ll T_{\gamma}$ the particle number density will be $\propto T^{4}$, but the density will be frozen when the annihilation rate $\chi \bar{\chi} \rightarrow \gamma \gamma$ is equal to the Hubble constant. Therefore we can determine the annihilation cross section that is required by the density of Eq. 2.1. Quantitatively for the WIMP model this cross section is estimated to be :

$$
\left\langle\sigma_{\chi \bar{\chi}} v\right\rangle_{T_{f}} \simeq 3 \times 10^{-26}\left(\frac{\rho_{\chi}}{3 \times 10^{-6} \mathrm{GeV} / \mathrm{cm}^{3}}\right) \mathrm{cm}^{3} \mathrm{~s}
$$

where $T_{f} \simeq m_{\chi} / 22.6$ [6]. It is remarkable that from the CMBR fit of Eq. 2.1 this cross section is in the scale of weak interaction.

An additional input from the thermal annihilation cross section of the WIMPs can be derived from the Big Bang Nucleosynthesis, which is obviously affected by the annihilation of WIMPs into into ordinary matter, causing a delayed injection of nucleons (and anti-nucleons) that could increase the ratio neutron to proton. From the estimated abundance of primordial deuterium is possible to derive the limits to the annihilation cross in function of the WIMP mass, shown as blue exclusion plots in Fig. 2 [7].

Some indirect indications on the physics of the CDM comes from the cosmological large scale distribution, that recently has been studied with the gravitational lensing of the light from distant galaxies. The area of the sky at high galactic latitude explored by the Dark Energy Survey (DES) experiment is of $\sim 250 \mathrm{deg}^{2}$ in which about 1 million galaxies at a distance of $0.6 \leq z_{G} \leq 1.2$ (an approximated average distance of $3 \mathrm{Gpc}$ ). The mass map obtained by the light shear shows a significant correlation at $6.8 \sigma$ 's with the distribution of the cluster of galaxies, only if smoothed with a Gaussian spread function of width 20 arcmin, decisively larger then the resolution of the survey $\left(\sim 5 \times 5 \operatorname{arcmin}^{2}\right)[8,9]$. The smoothing of the mass on scales of $\sim 10 \mathrm{Mpc}$ is difficult to reconcile simply scaling the profile of the hot intracluster gas that emits in the soft X-rays [10]. A similar situation is found in the study of the rotation curves of spiral galaxies [11] that shows a substantial flattening for distances $\sim 10 \mathrm{kpc}$ from the center. The pure Newtonian dynamics of the rotation of the luminous matter indicates that gravity field is produced by a sphere of uniform density, and not by a self gravitating ensemble of non-collision particles [12]. A possible explanation of this fact could be the existence of a large mass of dark baryon in the inner part of the spiral galaxies[13], but alternatively it could be also the indication of self-interaction of the DM particles[14]. In the latter case the original "cuspy" halos could have been smoothed out, on a scale equal to the free streaming distance. It is puzzling that the scale of the observed smoothing is in the range of few Mpc's at maximum, while for WIMP's should be much larger then the size of the Universe. In fact, if the smoothing of the galactic halos is due to elastic self-interaction of the DM particles, the cross section should be very high, of the order of $\left.\sigma / m \approx(0.8-10.) \times 10^{-24} \mathrm{~cm}^{2} / \mathrm{GeV} / \mathrm{c}^{2}\right)^{-1}[15]$. 


\section{Dark Matter and Astrophysics}

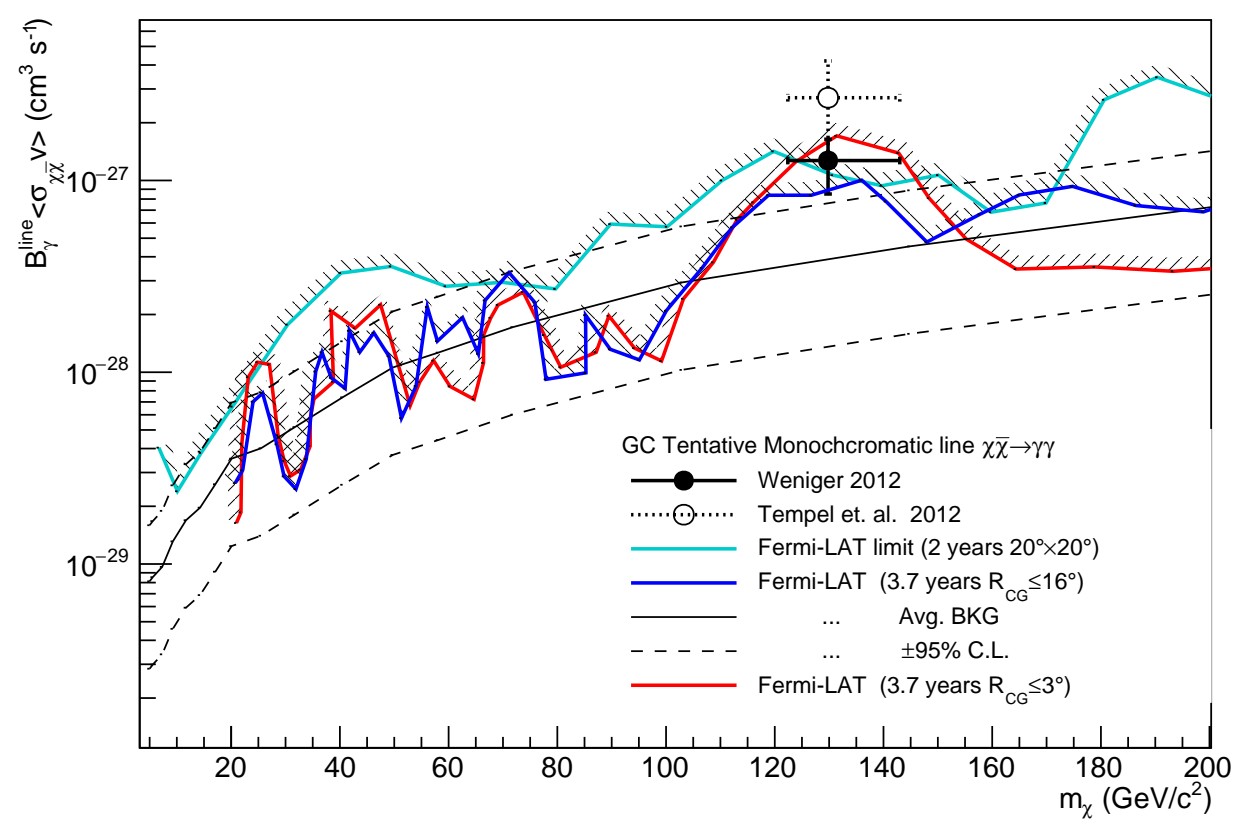

Figure 2: The complex situation of the possible $\gamma$-ray line at $130 \mathrm{GeV}$ from $\mathrm{Sgr} A^{*}$. The Fermi-LAT Collaboration limits are respectively from Ref.'s [16] and [17]. The absolute values of the line cross section, as well as the UL, is calculated with a template HM. For different HM's it could be higher by a factor $\sim 2$.

Annihilation of DM into ordinary matter should be astronomically detectable in the High Energy $\gamma$-ray band[18, 19]. The ideal channel for this observation is the process $\chi \bar{\chi} \rightarrow \gamma \gamma$ (or $\chi \bar{\chi} \rightarrow \gamma v$ ) because this reaction originates a monochromatic emission at $E_{\gamma}=m_{\chi} / 2$, easy to distinguish from the SM astrophysical production. In 2012 there was a considerable excitement caused by the possible detection in the public data collected by the Large Area Telescope for $\gamma_{\text {rays }}$ on the Fermi Gamma Ray Satellite (Fermi-LAT) of a sharp excess at $E_{\gamma} \simeq 130 \mathrm{GeV}$, from the direction of the very powerful astronomical radio-source $\operatorname{Sgr} A^{*}$ in the center of the Milky Way, [20]. In Fig. 2 we show this as a point on the $m_{\chi}$ vs. $\left\langle\mathscr{B}_{\gamma}^{\text {line }} \sigma_{\chi \bar{\chi}} v\right\rangle$. The presumable flux of the $\gamma$-ray line signal is obtained from the counting rate ${ }^{1}$. The annihilation cross section is derived from the equation

$$
\phi_{\gamma}^{\text {line }}=\frac{\left\langle\mathscr{B}_{\gamma}^{\text {line }} \sigma_{\chi \bar{\chi}} v\right\rangle \rho_{\odot}^{2} J_{H M}(\Delta \Omega)}{8 m_{\chi}^{2}}
$$

where $\left\langle\sigma_{\chi \bar{\chi}} v\right\rangle$ is the total annihilation cross section, $\mathscr{B}_{\gamma}^{\text {line }}$ the (unknown) branching ratio to the monochromatic channel, $\Delta \Omega$ the solid angle of the region of interest, and $J_{H M}$ the boosting factor $\propto\left\langle n_{\chi}^{2}\right\rangle_{H M}$ calculated on the assumed halo model (HM) for the inner part of the DM galactic halo. In facts the absolute evaluation of the cross section is strongly dependent from the halo model if

\footnotetext{
${ }^{1}$ For a full description of the complex technical and statistical methods of the $\gamma$-ray flux signal extraction see the references of each experiments.
} 
$\Delta \Omega \leq 20^{\circ} \times 20^{\circ}$ [21]. In Fig. 2 we report also the result of an independent analysis of the same data, that confirms the $\gamma$ line-like feature, that from the distribution in galactic coordinates of the pulses observe that the possible emission comes from a very thin disk of $\Delta b_{G} \sim \pm 0.5^{\circ}$ and width $\Delta \ell_{G} \sim$ $\pm 50^{\circ}$, well aligned with the galactic plane[22]. On the contrary, in a paper published immediately after those two, the Fermi-LAT collaboration published an analysis, largely overlapping the same data sample, that excluded at $95 \%$ C.L. the presence of any $\gamma$-ray line, in the interval $4 \leq E_{\gamma} \leq 200$ $\mathrm{GeV}$ from a region of $20 \times 20 \mathrm{deg}^{2}$ around the Galactic Center [16]. The upper limit (UL) to the cross section with 95\% CL is shown in Fig. 2 by the violet exclusion line. More recently the Fermi-LAT Collaboration have officially presented the results of the search for $\gamma$-ray lines in 3.7 years of exposure of the telescope[23]. The blue exclusion line in Fig. 2 shows the limit given by the investigation of the $\gamma$-ray detected in a cone of opening $16^{\circ}$ in the direction of the Galactic Center, while the red one is for a cone of $3^{\circ}$. The comparison of the two latter curves suggest that the $130 \mathrm{GeV}$ line is marginally compatible with the best Fermi-LAT limit for a compact source in the Galactic Center with a radius $\sim 0.5 \mathrm{kpc}[17]$.

The inclusive channel annihilation $\chi \bar{\chi} \rightarrow \gamma X$ is in principle detectable from the $\gamma$-ray broad band spectrum. In general we expect, from a naï ¿œve fireball picture, the equal production of pairs of all species of particles, including the SM ones, with thermal distribution with the only constraint of the energy conservation. In the cooling phase, the SM particle's pairs originate showers $\chi \bar{\chi} \rightarrow$ $\left(q \bar{q}, g g, \ell \bar{\ell}, \gamma^{*} X\right) \rightarrow \gamma X$, that can be modeled with classical HEP MC codes[24].

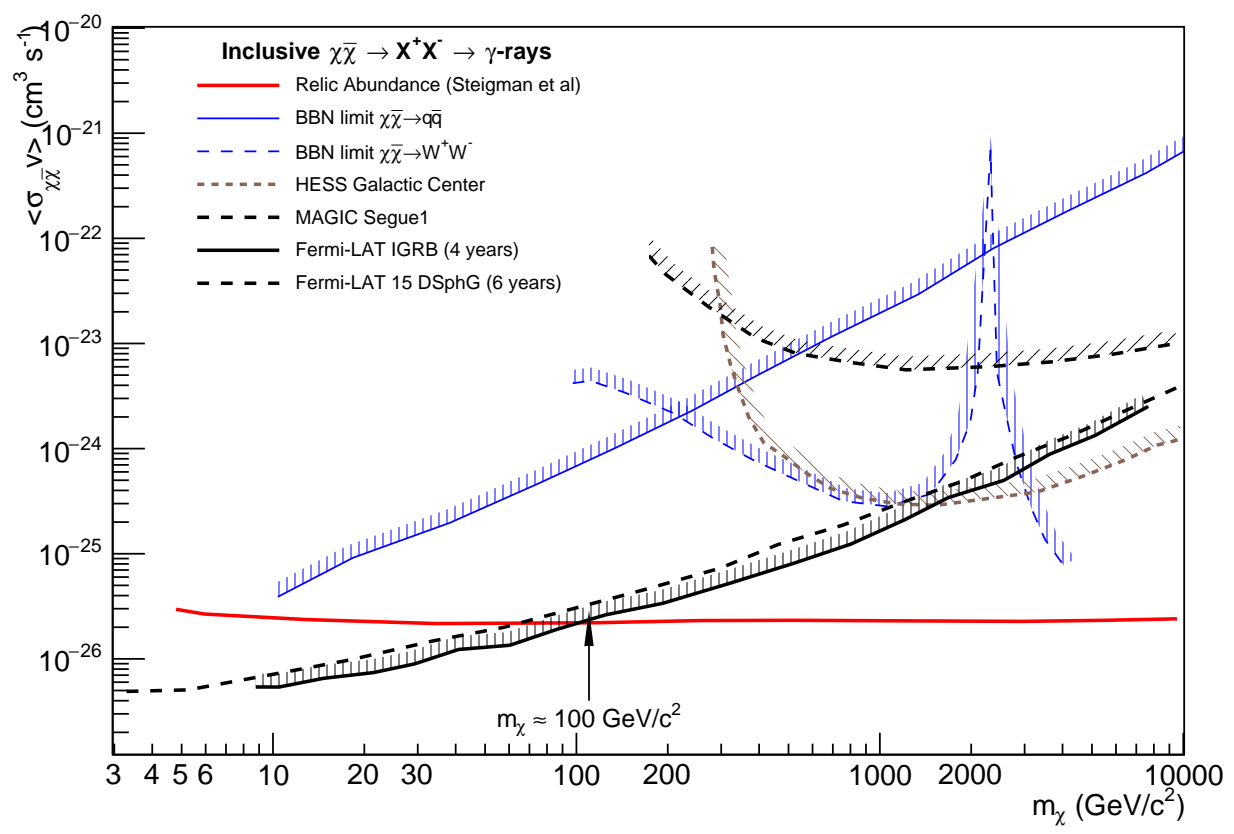

Figure 3: DM limits derived from the annihilation into the $\gamma$-ray inclusive channel (see text).

In Fig. 3 we show a partial compilation of the search for inclusive $\gamma$-rays production. The dotted exclusion curve is due to the HESS Collaboration[25] extrapolated from the emission in the inner central section of the Milky Way. The dot-dashed exclusion curves have been obtained by 
the MAGIC Collaboration from the faint spherical object Segue1[26] that is s very likely a dwarf satellite galaxy of the Milky Way, at $23 \mathrm{kpc}$ in the Leo constellation. This very peculiar object is a good candidate for the search of DM annihilation, because it shows a ratio of mass to luminosity of 3400 solar units[27]. However, the most interesting limit, among that shown in Fig. 3, the most interesting is the result on the Isotropic Gamma Ray Background (IGRB) of the Fermi-LAT Collaboration[28]. In this study $\gamma$-rays from a number of high galactic latitude regions of the sky were collected for 4 years. In this case the upper limit is derived from the average of a large number of galactic DM halos, that at least in principle should be closer to the real cosmological density. It is evident that from this experiment we should exclude masses for DM particles below $100 \mathrm{GeV} / \mathrm{c}^{2}$. It is to be remarked, however, that the limits discussed above can be applied only if DM and anti-DM have about the same abundance in the present Universe. If by chance the DM is asymmetric, as the baryons are, the $\gamma$-ray emission would be suppressed by a factor $\sim \rho_{\bar{\chi}} / \rho_{\chi}$, being definitively undetectable.

\section{Dark Matter direct search}

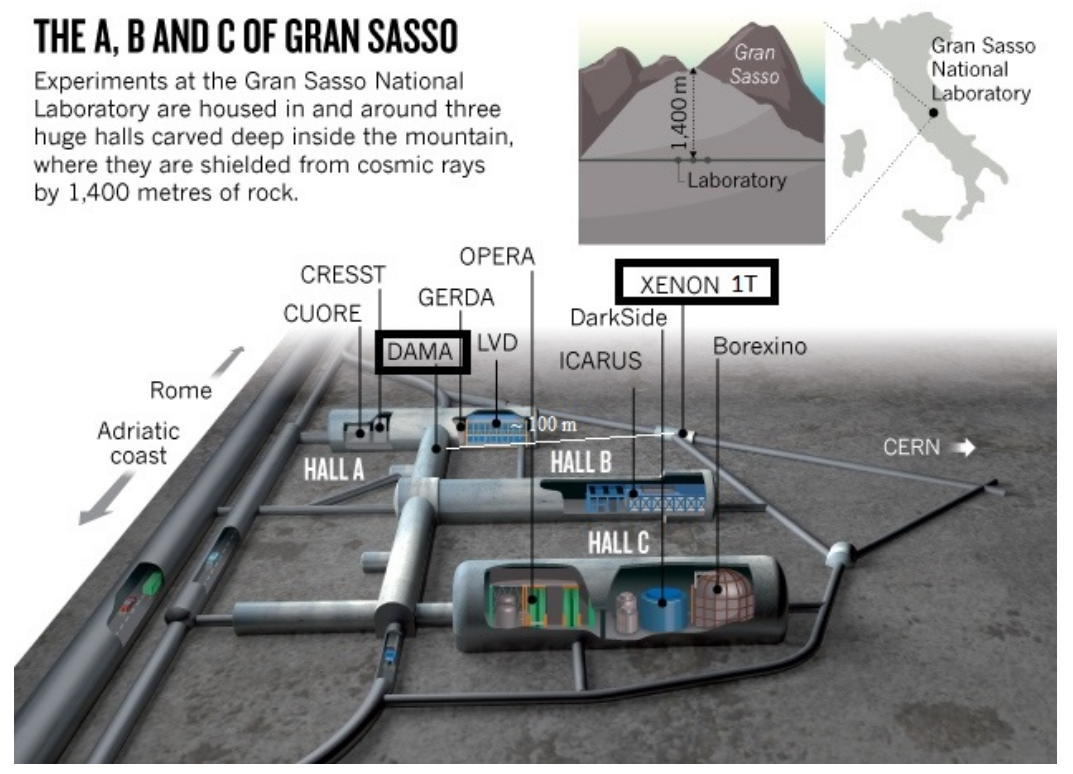

Figure 4: A visual evidence of the DM direct detection dispute. The two conflicting experiments on the direct detection of DM (namely D.A.M.A/LIBRA and Xenon1T) are both located in the LNGS-INFN laboratory of Assergi, Italy, only 100 meters one from the other.

The only claim of signal for DM recoiling has been advanced by the DA.MA. detector at the INFN Gran Sasso underground laboratory. The search for DM with highly radio-pure NaI(Tl) scintillating crystal has been started Gran Sasso about 20 years ago[29]. The scintillation light produced by of an hypothetical WIMP depends from the number of ionization pairs produced by a very low energy recoil nuclei. The recoil energy of the nucleus is 


$$
E_{r e c}=E_{\chi} \frac{2 m_{\chi}^{2}}{\left(m_{\chi}+m_{A}\right)^{2}} \sin ^{2} \frac{\theta}{2}
$$

where $m_{A}$ is the mass of the recoiling nucleus ${ }^{2}, E_{\chi} \simeq \frac{1}{2} m_{\chi} v_{\text {halo }}^{2}$ the kinetic energy of the WIMP and $\theta$ the c.m.s. scattering angle. With $v_{\text {halo }} \simeq 300 \mathrm{~km} / \mathrm{s}$ and an eventual WIMP mass $m_{\chi} \sim 10$ $\mathrm{GeV} / \mathrm{c}^{2}$ scatters $\left(84.7 \%\right.$ of the times) with the iodine $\left(A_{I} \simeq 129\right)$ with an average recoil energy $\left\langle E_{\text {rec }}\right\rangle_{I} \simeq 25.9 \mathrm{keV}$

The first claim of the apparent detection of nuclear recoils form DM has been given by the DA.MA./NaI(Tl) detector, an assembly of $100 \mathrm{~kg}$ of scintillating crystals, carefully shielded from natural radioactivity and cosmic ray in the Gran Sasso Laboratory in 1999[30]. Further observations obtained by the $250 \mathrm{~kg}$ DAMA/LIBRA detector in the same location, are proposed as the possible detection of a time dependent light production compatible with the DM recoil of nuclei[31]. Since that time, the modulated signal has been observed by the DAMA collaboration for 14 annual cycles (total exposure 1.36 ton $\times$ years), reaching a 9.3 $\sigma$ 's statistical C.L. [32]. The mass of the hypothetical WIMPs is in the range $7 \leq m_{\chi} \leq 50 \mathrm{GeV} / c^{2}$ and the cross section $\left(10 \geq \sigma_{\chi N a I(T l)} \leq 1\right) \times 10^{-41} \mathrm{~cm}^{2}(90 \%$ C.L.). The error bands are propagation of the systematic uncertainty on the calibration of the scintillation yield of the crystals[33, 34] and of the halo model[35] .A possible confirmation of the same effect has been claimed by the CoGeNT collaboration, using a single $440 \mathrm{~g}$ high-purity germanium crystal cooled to liquid nitrogen temperatures[36], in Soudan Underground Laboratory, MN, USA. The charge collection electronics of this experiment has detected in 3 years data taking (total exposure $1.3 \mathrm{~kg}$ years) at $2.2 \sigma$ 's statistical significance a modulation signal compatible in periodicity and phase to the DAMA one[37]. In this case the fitted hypothetical WIMP should have $6.5 \leq m_{\chi} \leq 9.5 \mathrm{GeV} / \mathrm{c}^{2}$ and $\left(5.9 \geq \sigma_{\chi G e}^{S I} \geq 2.1\right) \times 10^{-41}$ $\mathrm{cm}^{2}(90 \%$ C.L.).

The cryogenic technique has been proposed in the early 80's by Drukier \& Stodolsky[38] for the detection of cosmic neutrinos. The scattering media in this type of detectors is the liquid Xenon $\left(A_{X e} \simeq 131\right)$ and the recoil ionization can be detected by scintillation and/or charge collection. The Xenon100 detector, a combination of TPC for the detection of the ionization and of PMT's for the detection of the scintillation light, has been constructed at the LNGS starting from 2007[39]. One advantage of this technique is the possible accurate measurement of the rise time of the pulse of charge, that allows a discrimination of long tracks from the short tracks, expected in case of nuclear recoils[40]. After 100 days of data taking (exposure of $17 \mathrm{~kg} \times$ year), this experiment gave an upper limit to the spin-independent cross section $\sigma_{\chi X e}^{S I} \leq 6 \times 10^{-42} \mathrm{~cm}^{2}$ at $80 \%$ C.L.[41] in the same mass range of the claimed signal. A search for a modulated signal with an annual periodicity was shown to have a probability $P_{365} \leq 0.6 \%$, but an annual signal with the DAMA phase has been found to be excluded at $4.8 \sigma$ level [42].

More recently the XENON1T Collaboration has published the first results of the liquid xenon detector at Gran Sasso, after its upgrading to the fiduciary mass of $1042 \mathrm{~kg}$. In 32.4 days of data taking this detector has pushed the limit on cross section to $\sigma_{\chi X e}^{S I}\left(m_{\chi}=35 \mathrm{GeV} / c^{2}\right) \leq 7.7 \times 10^{-47}$

\footnotetext{
${ }^{2}$ We remind that the binding energy of nuclei are in the range of the MeV's. On the other side the de Broglie wavelength of a $10 \mathrm{GeV} / \mathrm{c}^{2}$ mass particle at $\beta \sim 10^{-3}$ is about $30 \mathrm{fm}$, larger than size of the molecules
} 
$\mathrm{cm}^{2}$ (90\% C.L.) [43]. A compatible limit $\sigma_{\chi X e}^{S I}\left(m_{\chi}=50 \mathrm{GeV} / \mathrm{c}^{2}\right) \leq 2.2 \times 10^{-46} \mathrm{~cm}^{2}$ has been obtained from the Large Underground Xenon (LUX) detector [44] at Soudan.

Table 1: Estimate of relative detection rates for different type of target media[45]

\begin{tabular}{|c|c|c|c|c|c|c|c|}
\hline & & $\mathrm{A}$ & $\mathrm{Z}$ & $\mathrm{f}_{\text {coll }^{a}}$ & $\mathrm{Q}_{\text {ion }}{ }^{b}$ & $\mathrm{R}_{10 \mathrm{GeV} / c^{2}}$ & $\mathrm{R}_{100 \mathrm{GeV} / c^{2}}$ \\
\hline \hline \multirow{2}{*}{$\mathrm{NaI}$} & $\mathrm{Na}$ & 22.99 & 11 & $15.3 \%$ & 0.2 & \multirow{2}{*}{2.14} & \multirow{2}{*}{123} \\
\cline { 2 - 8 } & $\mathrm{I}$ & 126.90 & 53 & $84.7 \%$ & 0.15 & & \\
\hline \multicolumn{2}{|c|}{$\mathrm{Si}$} & 28.09 & 14 & $100 \%$ & 0.21 & 12.2 & 21.2 \\
\hline \multicolumn{2}{|c|}{$\mathrm{Ge}$} & 72.61 & 32 & $100 \%$ & 0.17 & 4.07 & 81.3 \\
\hline \multicolumn{2}{|c|}{$\mathrm{Xe}$} & 131.29 & 54 & $100 \%$ & 0.15 & 0.32 & 147 \\
\hline
\end{tabular}

${ }^{a}$ Fraction of collisions

${ }^{b}$ Quenching efficiency averaged over the halo velocity distribution.

Many authors have proposed ways in which the difference about the two type of detectors could justify the discrepancy (for a review see Ref. [46]). As a matter of fact Eq. 4.1 shows that the recoil energy depends strongly from the atomic mass of the target nuclei. In Table 1 we show a theoretical evaluation of the relative rate (in arbitrary units) expected for the different type of detectors[45], if we assume the same numerical halo model[47]. From this table we can see that the most interesting scattering material for low mass WIMPs $\left(10 \mathrm{GeV} / \mathrm{c}^{2}\right.$ or less $)$ is the silicon. This material has been used in the SuperCDMS detector at Soudan. The most recent limit set by this $\sim 660 \mathrm{~g}$ experiment (total exposure of $1690 \mathrm{~kg}$ days) for a steady signal is at the moment about $\sigma_{\chi S i}^{S I} \leq 1.4 \times 10^{-44} \mathrm{~cm}^{2}$ for $m_{\chi} \geq 12 \mathrm{GeV} / \mathrm{c}^{2}$ [48]. Tucker-Smith \& Weiner [49] have observed that the inelastic scattering of a WIMP on a nuclei is kinetically allowed only if

$$
E_{\chi}^{\min } \geq \delta\left(1+\frac{m_{A}}{m_{\chi}}\right)
$$

where $\delta$ is energy lost in the collision. Calculations show that inelasticity of $\delta \sim 15 \mathrm{keV}$ would give a signal detectable in DAMA but not in the others experiments[50]. One way to check this hypothesis is to look at the recoil energy distribution, that should be depressed at low energies. A specific reanalysis of the XENON100 data shows that for $m_{\chi} \geq 50 \mathrm{GeV} / \mathrm{c}^{2}$ is $\delta<100 \mathrm{keV}$ [51].

\section{Dark matter and Particle Physics}

The main objective of the CERN Large Hadron Collider, an accelerator that produces high energy $p p$ collisions approved by CERN Council in December 1994, has been the search for the Higg particle, which was at the time the only missing prediction of the Standard Model (SM). LHC started the first Research Run I on 30 March 2010 at $\sqrt{s}=7 \mathrm{TeV}$. In 2012 has been found a particle with a mass of $125.09 \mathrm{GeV} / \mathrm{c}^{2}$, that is very likely the Higgs boson. After the final confirmation of the SM as an extremely accurate description of the sub-nuclear physics, the HEP community considers of extremely importance to investigate possible limitation of the SM, by searching evidence for deviations from its predictions, that are indicated generically as "New Physics" (NP). 


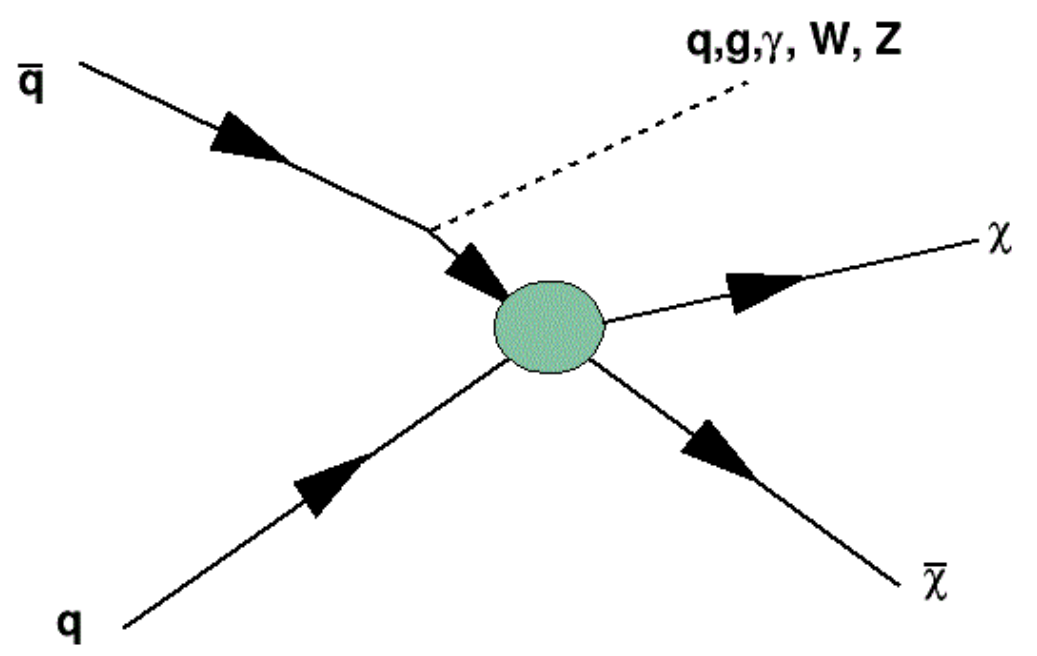

Figure 5: Missing energy search for DM at LHC

One indication in this direction could be the identification of heavy neutral particles with mass $m_{\chi} \geq 5-10 \mathrm{GeV} / \mathrm{c}^{2}$, that could form the DM observed in the Universe. One simple way of detect those particles is the search for events of the type $p p \rightarrow X+$ anything[52], where $X$ is a hadronic jet, or $\gamma$ or $W / Z$, with very high transverse momentum (usually $p_{T} \geq 100 \mathrm{GeV} / \mathrm{c}$ ). The missing energy of the event, defined

$$
E_{T}^{m i s s}=-\sum_{j \neq X} \vec{p}_{j} \sin \theta_{j}
$$

could be due to an undetected WIMP. The signal of NP would be a statistically significant number of events with $E_{T}^{\text {miss }} \geq 300 \mathrm{GeV}$ in excess over the SM background calculated with Monte Carlo simulation and validated on the same data [53]. The search for a missing energy signal, compatible with the DM production from $p p$ collisions is conducted at LHC since the starting of scientific runs in 2010, without any positive detection. Recently the ATLAS experiment has published the best upper limit of an analysis of $36.1 \mathrm{fbarn}^{-1}$ in Run 2 at $\sqrt{s}=13 \mathrm{TeV}$ [54]. The upper limit to the cross section is $A \times \varepsilon \times \sigma_{p p \rightarrow \text { Jet }+X} \leq 531$ fbarn at $95 \%$ C.L., for $p_{T}^{\text {Jet }} \geq 100 \mathrm{GeV}$ and $E_{T}^{\text {miss }} \geq 350$ $\mathrm{GeV}$. 


\section{s- channel}

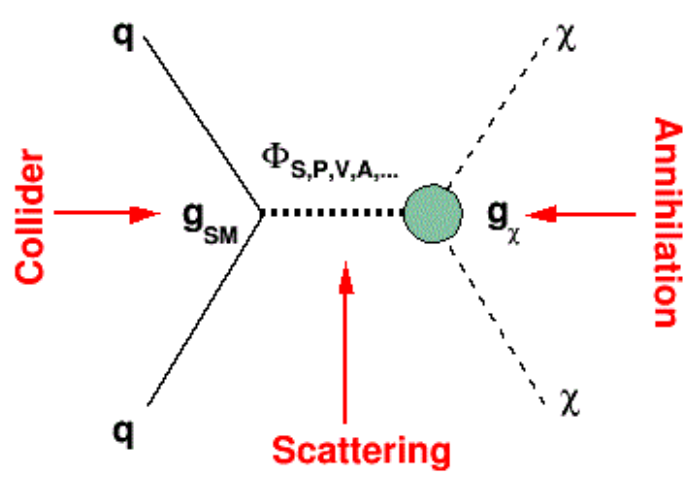

Figure 6: Schematic representation of an EFT process.

A generic interaction of the DM with the ordinary matter can be represented phenomenological by an Effective Field Theory (EFT) model[55, 56, 57], schematized in the Feynman graph shown in Fig. 6. The interaction Lagrangian of the process $f \bar{f} \rightarrow \phi \rightarrow \chi \bar{\chi}$

$$
\mathscr{L}_{i n t}=\frac{g_{\chi} g_{q}}{M_{*}^{2}}\left(f O_{S M} \bar{f}\right)\left(\chi \mathscr{O}_{\chi} \bar{\chi}\right)
$$

depends from four free parameters : the mass $m_{\chi}$ of the DM particle, the mass $M_{*}$ of the mediator (often called "portal" in the literature), and the two coupling constants $g_{q}$ and $g_{\chi}$ of the two vertices. The cross section for jets plus missing energy events at LHC, for a non-conservative interaction model, can be approximated[56, 57]:

$$
\sigma_{p p \rightarrow J e t+E_{T}^{m i s s}} \simeq \frac{\alpha_{s}}{32 \pi} N_{C} \frac{g_{\chi}^{2} g_{S M}^{2}}{M_{*}^{4}} S \mathscr{F}_{p p} \simeq 20\left(\frac{\sqrt{s}}{13 \mathrm{TeV}}\right)^{2}\left(\frac{g_{\chi} g_{q}}{0.25}\right)^{2}\left(\frac{1 \mathrm{TeV} / c^{2}}{M_{Z}}\right)^{4} \text { fbarn }
$$

where $\alpha_{S}$ is the strong coupling constant, $N_{C}$ the number of QCD colors and $\mathscr{F}_{p p}$ is the average fraction of $p p$ c.m.s. energy communicated to the interacting quarks pair, calculated from the partonic distribution function (PDF) ${ }^{3}$. The limit to the production efficiency of WIMPs in the LHC Run 2 from the ATLAS and CMS experiments is represented in the exclusion plot of $m_{\chi}$ vs. $M_{S}$ reported in Fig. 7. In the figure is indicated the energy conservation limit $m_{\chi} \leq M_{*} / 2$, that excludes (for the benchmark model) any mass of WIMP $m_{\chi} \leq 100 \mathrm{GeV} / \mathrm{c}^{2}$. However it is evident from Fig. 7 that the mass of a DM particle that could be compatible with the estimated primordial annihilation cross section should be $m_{\chi} \geq 430_{-20}^{+10} \mathrm{GeV} / \mathrm{c}^{2}$.

\footnotetext{
${ }^{3}$ The numerical value is applicable to the benchmark model of Dirac DM and vector-axial coupling in the $s$-channel. More conservative models predict smaller cross sections (see Ref. [57])
} 


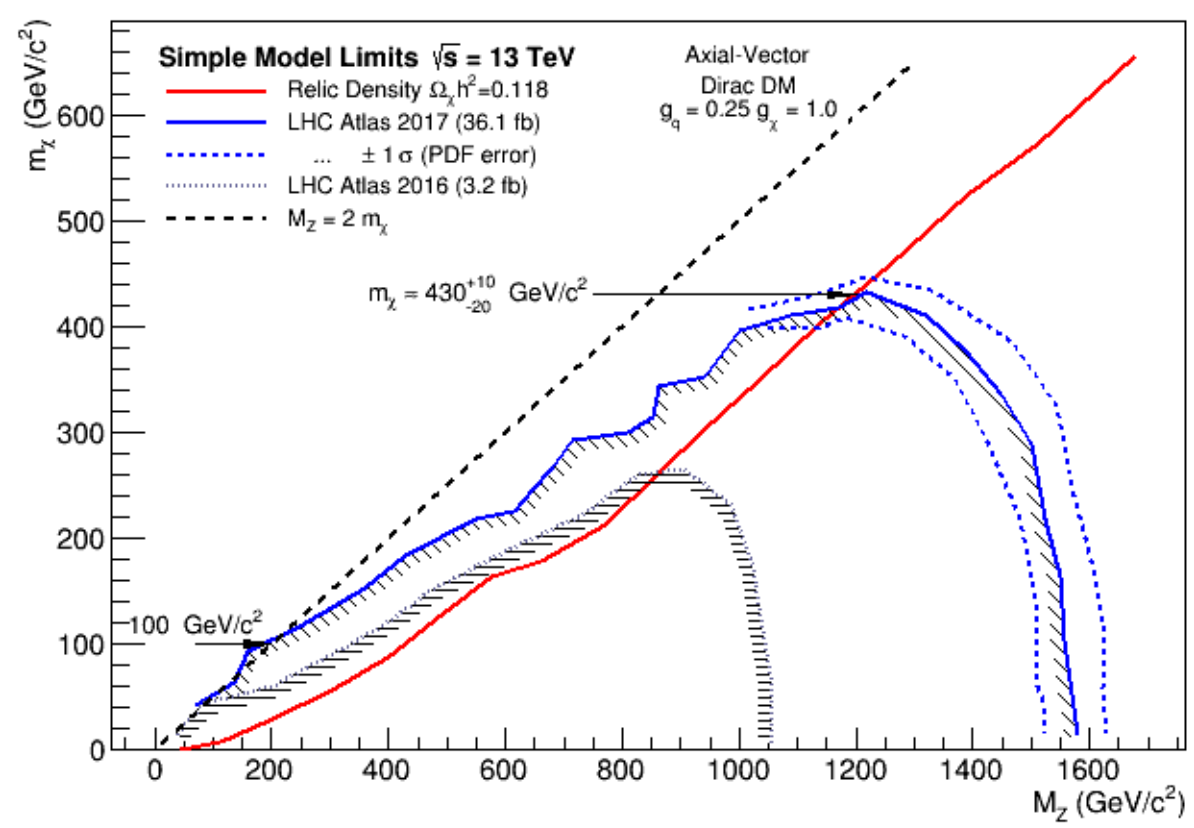

Figure 7: The best limits from LHC derived from the benchmark EFT model of WIMP nucleon interaction (Adapted from Ref.'s [53, 58]) (see text).

Reversed from right to left the the Feynman diagram of Figt. 6 represents the annihilation reaction, for which the benchmark model predicts a cross section [59]

$$
\sigma_{a n n} v_{f} \simeq \frac{24 N_{C}}{\pi} \frac{g_{\chi}^{2} g_{S M}^{2}}{M_{*}^{4}} m_{\chi}^{2} \simeq 2.5 \times 10^{-26}\left(\frac{m_{\chi}}{10 \mathrm{GeV} / \mathrm{c}^{2}}\right)^{2}\left(\frac{g_{q} g_{\chi}}{0.25}\right)^{2}\left(\frac{1 \mathrm{TeV} / \mathrm{c}^{2}}{M_{*}}\right)^{4} \mathrm{~cm}^{3} s^{-1}
$$

The solid red-line shown in Fig. 7 is the $m_{\chi}$ vs. $M_{*}$ that is obtained from the Eq. 5.4 for the measured relic DM abundance, under the assumption that the freeze out temperature of cosmic $\mathrm{DM}$ is $T_{f} \simeq m_{\chi} / 22$ [60]. It is worth noticing that the thermal anrnihilation rate required in order to justify the DM relic abundance is excluded by the benchmark model applied to the $95 \%$ limit given by LHC for masses $m_{\chi} \leq 420 \mathrm{GeV} / \mathrm{c}^{2}$.

In the vertical direction the diagram of Fig. 6 represents the two-body elastic scattering $\chi \mathscr{N} \rightarrow \chi \mathscr{N}$ of the direct searches, which can be approximated [61]:

$$
\sigma_{\chi \mathscr{N}}^{S I} \approx \frac{9}{\pi} N_{C} \frac{g_{q}^{2} g_{\chi}^{2}}{M_{*}^{4}} \mu_{\chi \mathscr{N}}^{2} \simeq 0.24 \times 10^{-41}\left(\frac{g_{q} g_{\chi}}{0.25}\right)^{2}\left(\frac{1 \mathrm{TeV} / c^{2}}{M_{*}}\right)^{4} \mathrm{~cm}^{2}
$$

where and $\mu_{\chi \mathscr{N}}=m_{\chi} m_{N} /\left(m_{\chi}+m_{N}\right)$ is the reduced mass. 


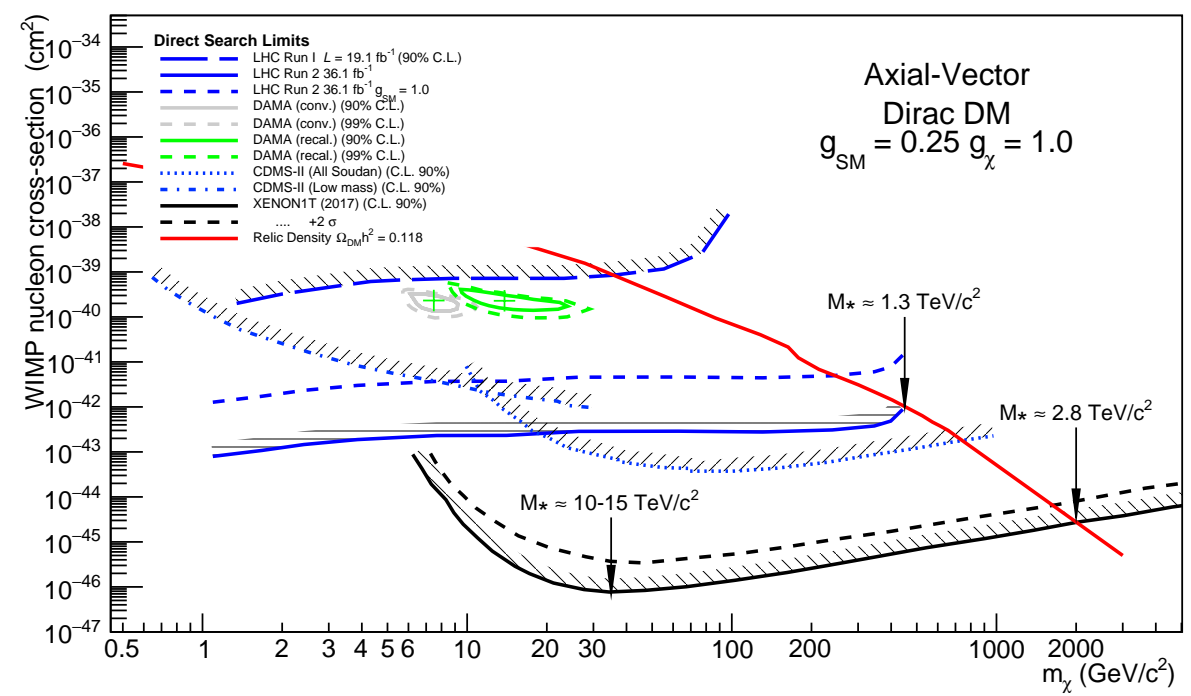

Figure 8: Comparison of the results of direct search for DM and the extrapolation of the spin-independent elastic cross section derived from the LHC limits on DM production, derived from the Simplified Model (see text).

On Fig. 8 we observe that the DAMA/LIBRA signal, that was compatible with the LHC data at $3.2 \mathrm{fb}^{-1}$, is now excluded by the 10 times larger statistics, collected until now. Moreover the limit to the scattering cross section set by the XENON1T experiment would exclude masses $m_{\chi} \leq 2$ $\mathrm{TeV} / \mathrm{c}^{2}$, at least according to the benchmark model. If this is true the production cross section of WIMPs at LHC can be still of the order of 5-10 fb, detectable with the upgrade of the LHC collider to the High Luminosity LHC, with an increase of the luminosity by a factor 10, expected to be operational in 2025.

However the impression that we have from the experiments is that the physical nature of dark matter is in this moment particularly oscure! The only clear information that we have about DM is that very likely it is there and interacts gravitationally with the ordinary matter. If this would be the only type of interaction of the DM ...

\section{Non-WIMP Dark Matter ?}

As we have seen in the $\S 1$ the principal scientific motivation of the WIMP model is given by the Eq. 2.2, namely the fact that the annihilation cross section predicted by cosmology for a production is in the weak scale. For the moment the only firm evidence on the nature of the DM particles that we have is that it couples with the ordinary matter via the gravitational field. But we have now a considerable amount of evidence any eventual non-gravitational interaction should be well above the EW scale of $\sim 100 \mathrm{GeV}$, at least by a factor of the order of 10 . Many alternative models for the DM have been proposed, but at the moment we do not have any experimental evidence supporting any one of these. But the main result of LHC until now is that the SM is the best description of the known experimental particle physics that we have at the moment. 

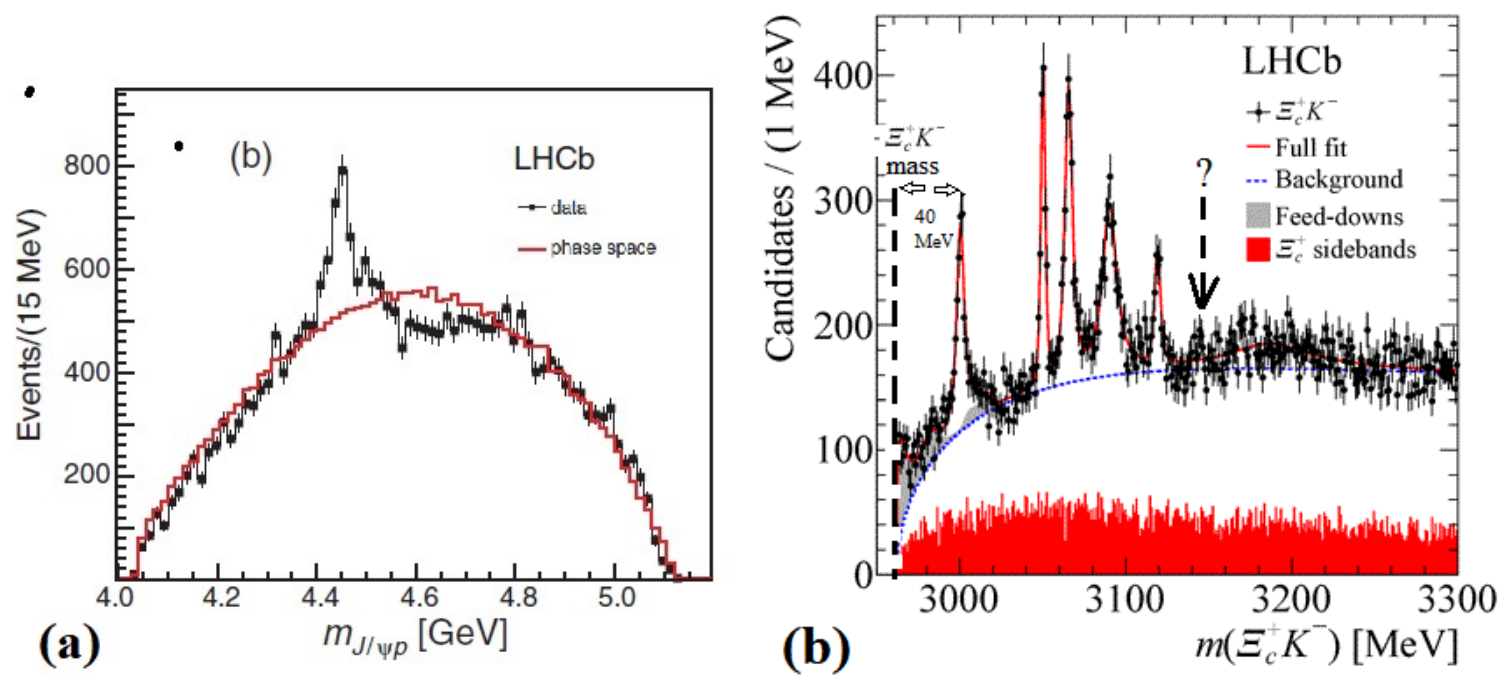

Figure 9: Heavy beauty resonances, with masses in the range 3-5 GeV/c $\mathrm{c}^{2}$, observed in the Run 1 of the LHCb detector. In panel (a) couple of particles that decay like pentaquarks states $c \bar{c} u u d$ [62] and (b) five (or six) particles that can be possibly interpreted as $s s c q \bar{q}$ state [63].

As it is well known the ordinary matter is composed essentially by the two lighter type of quarks, while experimentally do exist much heavier quarks. As expected $p p$ collisions with center of mass energy 7,8 and $13 \mathrm{TeV}$ at LHC produce a plethora of new hadronic particles containing heavy quarks. The LHCb experiment has detected novel heavy resonances that can be interpreted as multiquarks systems [64, 65].

In the SM the only constraint to the existence of a particle is integral or null electric charge and baryon (or lepton) number. It is not inconceivable that one or more of these systems could be stable, if the decay is forbidden by energy, flavor and baryon number conservation [66, 67]. In the early universe the heavy exotic hadron will be extremely abundant soon after the electroweak (EW) phase transition from quark-gluon plasma (QGP) to the hadron gas (HG), Therefore we can speculate that a population of dark baryons could could be present in the Universe today [68].

In case of baryon the mass is due to the Higgs mechanism, Therefore it would be null before the EW transition at $T_{U} \sim 140 \mathrm{GeV}$. Consequently the present abundance of the DM is not related to the freeze out, but to the mechanism of baryogenesis, and the relation between the present density of DM and its thermal self-annihilation cross section, at expressed by Eq. 2.2, does not hold. Moreover the effect of stable heavy baryons with mass $m_{B} \gtrsim 10 \mathrm{GeV} / \mathrm{c}^{2}$ on the BBN is determined essentially by the inelastic cross section of the heavy baryon with the deuterium nuclei for $T_{B} \leq 2.2$ $\mathrm{MeV}$. The $\mathrm{D} / \mathrm{H}$ abundance uncertainty sets a very weak limit to this cross section in the region of many barns[15, 69].

The magic of these elusive particles is that could not have be detected in the various DM experiments. Detection in direct search in underground experiments is obviously impossible ${ }^{4}$ and the indirect searches with $\gamma$-ray astronomy would be hampered by the baryonic asymmetry. Moreover

\footnotetext{
${ }^{4}$ The two-body scattering cross section for neutral heavy exotic baryons will be proportional to $\propto \mu_{\chi \mathscr{N}}^{2}$ the reduced mass. In pratice not very different from the neutron cross section for $m_{\chi} \gg m_{\mathscr{N}}$.
} 
this kind matter is difficult to detect as missing energy because can produce visible showers in the tracking detector.

\section{Summary}

The impression that we have from the experiments is that the physical nature of dark matter is in this moment particularly obscure! The only clear information that we have about DM is that very likely it is there and interacts gravitationally with the ordinary matter. If this is the only type of coupling with the ordinary matter or fields, the scattering cross section would be of the infinitesimal order of magnitude $O\left(m_{\chi}^{2} / M_{P}^{4}\right)$ with $M_{P}=1.22 \times 10^{19} \mathrm{GeV} / \mathrm{c}^{2}$ is the Planck mass [70].

The simple WIMP model of DM primordial production in the Hot Universe before the Baryogenesis appears to be at the moment seriously questionable, because both the limits from the FermiLAT gamma ray telescope and the HEP Collider, that are compatible only with a mass largely in excess of $100 \mathrm{GeV} / \mathrm{c}^{2}$ ( or $\sim 400 \mathrm{GeV} / \mathrm{c}^{2}$ for the compatibility of the LHC Run 2 upper limit with the WIMP's thermal production). From the XENON1T experiment data, applying the EFT model, is estimated that WIMPs with mass in the range $10-100 \mathrm{GeV} / \mathrm{c}^{2}$ cannot be observed in the present generation of colliders.

At the present the application of EFT model to the DM search experiments suggests that any kind of "new physics" (like strings, supersymmetry, extra dimensions, and other) if exists, is acting at at a much higher energy scale then the Higgs scale. A scale that according the direct search experiments could be of the order of $10 \mathrm{TeV}$ or more. It is very remarkable that all the HEP experiments, up to now, confirm the absolute quantitative reliability of the SM for energies below few TeV's, that could be a problem from the WIMP's model.

On the other side experiments at colliders, including the ones of LHC Run 1, show that heavy multiquark systems are possible, as predicted by the QCD. It is very likely that neutral heavy exotics with masses up to $\sim 100 \mathrm{GeV} / \mathrm{c}^{2}$ if stable could form a population of cold dark baryons in the present universe, as hypothesized many years ago.

\section{References}

[1] F. Zwicky, Die Rotverschiebung von extragalaktischen Nebeln, Helvetica Physica Acta 6 (1933) $110-127$.

[2] F. Zwicky, On the Masses of Nebulae and of Clusters of Nebulae, Astroph. J. 86 (Oct., 1937) 217.

[3] V. C. Rubin and W. K. Ford, Jr., Rotation of the Andromeda Nebula from a Spectroscopic Survey of Emission Regions, Astroph. J. 159 (Feb., 1970) 379.

[4] Planck collaboration, P. A. R. e. a. P. C. Ade, Planck 2015 results. XIII. Cosmological parameters, Astronomy \& Astrophys. 594 (2016) A13, [1502.01589].

[5] E. Calabrese, R. A. Hlozek, N. Battaglia, E. S. Battistelli, J. R. Bond, J. Chluba et al., Cosmological parameters from pre-planck cmb measurements, $1302.1841 \mathrm{v} 2$.

[6] G. Steigman, CMB Constraints On The Thermal WIMP Mass And Annihilation Cross Section, Phys. Rev. D91 (2015) 083538, [1502.01884].

[7] M. Kawasaki, K. Kohri, T. Moroi and Y. Takaesu, Revisiting Big-Bang Nucleosynthesis Constraints on Dark-Matter Annihilation, Phys. Lett. B751 (2015) 246-250, [1509. 03665$].$ 
[8] C. Chang, V. Vikram, B. Jain, D. Bacon, A. Amara, M. R. Becker et al., Wide-field lensing mass maps from des science verification data, $1505.01871 \mathrm{v} 2$.

[9] DES collaboration, V. Vikram et al., Wide-field lensing mass maps from Dark Energy Survey science verification data: Methodology and detailed analysis, Phys. Rev. D92 (2015) 022006, [1504.03002].

[10] J. E. Taylor, Dark matter halos from the inside out, $1008.4103 v 2$.

[11] P. Salucci, The distribution of dark matter in galaxies, in BEYOND 2003, Castle Ringberg, Tegernsee, Germany, June 9-14, 2003, pp. 613-626, 2003. astro-ph/0310376.

[12] A. Kamada, M. Kaplinghat, A. B. Pace and H.-B. Yu, How the Self-Interacting Dark Matter Model Explains the Diverse Galactic Rotation Curves, Phys. Rev. Lett. 119 (2017) 111102, [1611.02716].

[13] M. Persic, P. Salucci and F. Stel, The universal rotation curve of spiral galaxies: I. the dark matter connection, astro-ph/9506004v3.

[14] D. N. Spergel and P. J. Steinhardt, Observational evidence for selfinteracting cold dark matter, Phys. Rev. Lett. 84 (2000) 3760-3763, [astro-ph/9909386].

[15] B. D. Wandelt, R. Dave, G. R. Farrar, P. C. McGuire, D. N. Spergel and P. J. Steinhardt, Selfinteracting dark matter, in Sources and detection of dark matter and dark energy in the universe. Proceedings, 4th International Symposium, DM 2000, Marina del Rey, USA, February 23-25, 2000, pp. 263-274, 2000. astro-ph/0006344.

[16] M. Ackermann and others (Fermi-LAT Collaboration), Search for dark matter in gamma-ray lines and the inclusive photon spectrum, $1205.2739 \mathrm{v} 1$.

[17] G. A. Gomez-Vargas, M. A. Sanchez-Conde, J.-H. Huh, M. Peiro, F. Prada et al., Constraints on WIMP Annihilation for Contracted Dark Matter in the Inner Galaxy with the Fermi-LAT, 1308.3515.

[18] F. W. Stecker, Gamma-rays and neutrinos from dark matter, astro-ph/9607037v1.

[19] J. L. Feng, Dark Matter Candidates from Particle Physics and Methods of Detection, Ann.Rev.Astron.Astrophys. 48 (2010) 495-545, [1003.0904].

[20] C. Weniger, A Tentative Gamma-Ray Line from Dark Matter Annihilation at the Fermi Large Area Telescope, JCAP 1208 (2012) 007, [1204.2797].

[21] F. Nesti and P. Salucci, The Dark Matter halo of the Milky Way, AD 2013, JCAP 1307 (2013) 016, [1304.5127].

[22] E. Tempel, A. Hektor and M. Raidal, Fermi $130 \mathrm{GeV}$ gamma-ray excess and dark matter annihilation in sub-haloes and in the Galactic centre, JCAP 1209 (2012) 032, [1205.1045].

[23] COLlaboration FOR THE FERMi-LAT collaboration, M. Gustafsson, Fermi-LAT and the Gamma-Ray Line Search, 1310.2953.

[24] M. Cirelli, G. Corcella, A. Hektor, G. Hutsi, M. Kadastik, P. Panci et al., PPPC 4 DM ID: A Poor Particle Physicist Cookbook for Dark Matter Indirect Detection, JCAP 1103 (2011) 051 , [1012.4515].

[25] A. Abramowski and others (HESS Collaboration, Search for a dark matter annihilation signal from the galactic center halo with h.e.s.s., Physical Review Letters 106 (apr, 2011) . 
[26] J. Rico, M. Wood, A. Drlica-Wagner, J. Aleksi?, for the MAGIC Collaboration and the Fermi-LAT Collaboration, Limits to dark matter properties from a combined analysis of magic and fermi-lat observations of dwarf satellite galaxies, $1508.05827 \mathrm{v} 2$.

[27] J. D. Simon, M. Geha, Q. E. Minor, G. D. Martinez, E. N. Kirby, J. S. Bullock et al., A complete spectroscopic survey of the milky way satellite segue 1: The darkest galaxy, $1007.4198 \mathrm{v} 3$.

[28] FERMI-LAT collaboration, M. Ackermann et al., Limits on Dark Matter Annihilation Signals from the Fermi LAT 4-year Measurement of the Isotropic Gamma-Ray Background, JCAP 1509 (2015) 008, [1501.05464].

[29] P. Belli, R. Bernabei, F. Montecchia, W. di Nicolantonio, A. Incicchitti, D. Prosperi et al., The DAMA experiments at Gran Sasso., Phys. Rep. (1998) .

[30] R. Bernabei, P. Belli, R. Cerulli, F. Montecchia, M. Amato, G. Ignesti et al., Investigation on possible diurnal effects induced by dark matter particles., Nuovo Cimento A Serie 112 (Dec., 1999) 1541-1552.

[31] P. Belli, R. Bernabei, A. Bottino, F. Cappella, R. Cerulli et al., Observations of annual modulation in direct detection of relic particles and light neutralinos, Phys.Rev. D84 (2011) 055014, [1106. 4667 ].

[32] R. Bernabei et al., Results and perspectives of DAMA/LIBRA, in American Institute of Physics Conference Series, vol. 1894 of American Institute of Physics Conference Series, p. 020003, Oct., 2017. DOI.

[33] R. Bernabei, P. Belli, F. Cappella, V. Caracciolo, S. Castellano, R. Cerulli et al., Model independent result on possible diurnal effect in DAMA/LIBRA-phase1, European Physical Journal C 74 (Mar., 2014) 2827, [1403.4733].

[34] R. Bernabei, P. Belli, A. di Marco, A. D’Angelo, A. Incicchitti, F. Cappella et al., Investigation on possible systematics and side processes, International Journal of Modern Physics A 31 (Oct., 2016) 1642008.

[35] P. Belli, R. Cerulli, N. Fornengo and S. Scopel, Effect of the galactic halo modeling on the DAMA / NaI annual modulation result: an Extended analysis of the data for WIMPs with a purely spin independent coupling, Phys. Rev. D66 (2002) 043503, [hep-ph/ 0203242$].$

[36] CoGeNT collaboration, C. E. Aalseth et al., CoGeNT: A Search for Low-Mass Dark Matter using p-type Point Contact Germanium Detectors, Phys. Rev. D88 (2013) 012002, [1208 . 5737].

[37] CoGeNT collaboration, C. E. Aalseth et al., Search for An Annual Modulation in Three Years of CoGeNT Dark Matter Detector Data, 1401.3295.

[38] A. Drukier and L. Stodolski, Principles and applications of a neutral current detector for neutrino physics and astronomy, in Massive Neutrinos in Astrophysics and in Particle Physics (J. Tran Thanh Van and F. Vannucci, eds.), p. 395, 1984.

[39] E. Aprile, The XENON100 Dark Matter Experiment, in American Institute of Physics Conference Series (S. Khalil, ed.), vol. 1115 of American Institute of Physics Conference Series, pp. 355-360, Apr., 2009. DOI.

[40] XENON100 collaboration, E. Aprile et al., The neutron background of the XENON100 dark matter search experiment, J. Phys. G40 (2013) 115201, [1306.2303].

[41] E. Aprile, K. Arisaka, F. Arneodo, A. Askin, L. Baudis, A. Behrens et al., Dark Matter Results from 100 Live Days of XENON100 Data, Physical Review Letters 107 (Sept., 2011) 131302, [1104.2549]. 
[42] E. Aprile and others (The XENON Collaboration), Search for Event Rate Modulation in XENON100 Electronic Recoil Data, ArXiv e-prints (July, 2015), [1507.07748].

[43] XENON collaboration, E. Aprile and others (The XENON Collaboration), First Dark Matter Search Results from the XENON1T Experiment, 1705.06655.

[44] LUX collaboration, D. S. Akerib et al., Results from a search for dark matter in the complete LUX exposure, Phys. Rev. Lett. 118 (2017) 021303, [1608.07648].

[45] G. Auriemma, “To be published.".

[46] D. B. Cline, Recent results on the low mass dark matter WIMP controversy: 2011, ArXiv e-prints (Sept., 2011), [1109.1799].

[47] M. Hoeft, J. P. Mucket and S. Gottlober, Velocity dispersion profile in dark matter halos, Astrophys. J. 602 (2004) 162-169, [astro-ph/0311083].

[48] SuperCDMS collaboration, R. Agnese et al., Low-Mass Dark Matter Search with CDMSlite, Phys. Rev. D (2017) , [1707.01632].

[49] D. Tucker-Smith and N. Weiner, Inelastic dark matter, Phys. Rev. D64 (2001) 043502 , [hep-ph/0101138].

[50] D. Tucker-Smith and N. Weiner, Inelastic dark matter at DAMA, CDMS and future experiments, Nucl. Phys. Proc. Suppl. 124 (2003) 197-200, [a.stro-ph/ 0208403 ].

[51] E. Aprile et al., Implications on inelastic dark matter from 100 live days of XENON100 data, Phys. Rev. D 84 (Sept., 2011) 061101, [1104.3121].

[52] M. Beltran, D. Hooper, E. W. Kolb, Z. A. C. Krusberg and T. M. P. Tait, Maverick dark matter at colliders, JHEP 09 (2010) 037, [1002 . 4137].

[53] ATLAS collaboration, M. Aaboud and others (ATLAS Collaboration), Search for new phenomena in final states with an energetic jet and large missing transverse momentum in pp collisions at $\sqrt{s}=13$ ??TeV using the ATLAS detector, Phys. Rev. D94 (2016) 032005, [1604.07773].

[54] A. Collaboration, Search for dark matter and other new phenomena in events with an energetic jet and large missing transverse momentum using the atlas detector, $1711.03301 \mathrm{v} 2$.

[55] J. Alwall, P. Schuster and N. Toro, Simplified Models for a First Characterization of New Physics at the LHC, Phys. Rev. (2009) 075020, [0 810 . 3921].

[56] Y. Bai, P. J. Fox and R. Harnik, The Tevatron at the Frontier of Dark Matter Direct Detection, JHEP 1012 (2010) 048, [1 005.3797$].$

[57] D. Abercrombie, N. Akchurin, E. Akilli, J. A. Maestre, B. Allen, B. A. Gonzalez et al., Dark matter benchmark models for early lhc run-2 searches: Report of the atlas/cms dark matter forum, $1507.00966 \mathrm{v} 1$.

[58] ATLAS collaboration, M. Aaboud and others (ATLAS Collaboration), Search for new phenomena in events with a photon and missing transverse momentum in pp collisions at $\sqrt{s}=13 \mathrm{TeV}$ with the ATLAS detector, JHEP 06 (2016) 059, [1604.01306].

[59] A. Albert et al., Recommendations of the lhc dark matter working group: Comparing lhc searches for heavy mediators of dark matter production in visible and invisible decay channels, $1703.05703 \mathrm{v} 2$.

[60] G. Steigman, Primordial Nucleosynthesis in the Precision Cosmology Era, Ann. Rev. Nucl. Part. Sci. 57 (2007) 463-491, [0 712 . 1100 ]. 
[61] M. R. Buckley, D. Feld and D. Goncalves, Scalar simplified models for dark matter, 1410.6497v2.

[62] LHCB collaboration, R. Aaij et al., Observation of J/?p Resonances Consistent with Pentaquark States in $?_{b}^{0}$ ? J/? $K^{-}$p Decays, Phys. Rev. Lett. 115 (2015) 072001, [1507.03414].

[63] LHCB collaboration, R. Aaij et al., Observation of five new narrow $\Omega_{c}^{0}$ states decaying to $\Xi_{c}^{+} K^{-}$, Phys. Rev. Lett. 118 (2017) 182001, [1703.04639].

[64] G. A. Cowan, Exotic hadron spectroscopy at the lhcb experiment, $1610.04906 \mathrm{v} 1$.

[65] A. Ali, J. S. Lange and S. Stone, Exotics: Heavy pentaquarks and tetraquarks, $1706.00610 \mathrm{v} 2$.

[66] I. W. Stewart, M. E. Wessling and M. B. Wise, Stable heavy pentaquark states, hep-ph/0402076v1.

[67] S. H. Lee and S. Yasui, Stable multiquark states with heavy quarks in a diquark model, Eur. Phys. J. C64 (2009) 283-295, [0 901 .2977].

[68] X. Liang and A. Zhitnitsky, Axion field and the quark nugget's formation at the qcd phase transition, $1606.00435 \mathrm{v} 2$.

[69] R. H. Cyburt, B. D. Fields, V. Pavlidou and B. D. Wandelt, Constraining strong baryon dark matter interactions with primordial nucleosynthesis and cosmic rays, Phys. Rev. D65 (2002) 123503, [astro-ph/0203240].

[70] N. E. J. Bjerrum-Bohr, J. F. Donoghue, B. R. Holstein, L. Plante and P. Vanhove, Light-like scattering in quantum gravity, $1609.07477 \mathrm{v} 3$. 